Bioinspired, Biomimetic and Nanobiomaterials Volume 3 Issue BBN1

Atomic force microscopic study on lipid

bilayer nanoscale phase separation

Liu
Pages 58-62 http://dx.doi.org/10.1680/bbn.13.00021 Short Communication Received 06/09/2013

Published online 29/09/2013

Keywords: atomic force microscopy/bilaye

fluorescence indicator/lipid/microdomains/phase separation/

self-assembly

\title{
Atomic force microscopic study on lipid bilayer nanoscale phase separation
}

\section{Rui Liu*}

Joint Center for Artificial Photosynthesis, California Institute of Technology,

Pasadena, CA, USA

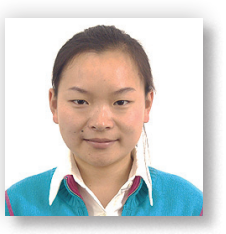

Phase separation of copolymers or lipid membranes in nanoscale has attracted increasing interests for their applications in the synthesis of inorganic nanomaterial. The nanoscale phase separation of liquid bilayer as a supported membrane is systematically investigated by atomic force microscope (AFM). Moreover, the position of the fluorescence indicator, which is commonly used in the phase separation study in optical microscope, is also probed in this study to complete the knowledge of tradition microscopy research. Overall, the authors demonstrate the advantages of using AFM for phase separation study in this article.

\section{Introduction}

Lipids bilayer phase separation is always a popular research field as a simplest mimic model for microdomains or rafts in biosystems, which are believed to play a significant role in protein sorting, signal transducting and cell growth regulating. ${ }^{1,2,3}$ It also develops researchers interested in the nanomaterial research recently for the self-assembly domain formation in nanoscale, which could be potentially used as nanomaterials' template in nanomaterials bottom-up synthesis methods. ${ }^{4,5}$

In the liquid crystal lipid domains, the soft hydrocarbon chain of the lipids generally is vertical to the surface of the membrane and their mobility is quite high, which allows their free movement inside of the membrane. In the solid state of the lipid domains, the rigid hydrocarbon chain would be vertical or shown a certain angle to the surface of the membrane. Typical phase separation occurs in the supported membrane formed with more than two lipid types. When the temperature is high enough, both types of liquids are soft and have high enough mobility in the membrane uniformly mixed together. With decreasing temperature, the mobility of the liquid is lowered until one of them achieved the solid phase. As a result, the phase separation is observed in normal cases. This process is studied in both optical microscope and atomic force microscope (AFM) by researchers. ${ }^{6,8}$ However, the drawback of optical microscope is more and more obvious for the limited observation resolution, lack of details and unavoidable introduction of the fluorescence indicator. AFM study could provide us more detailed information compared with optical microscope with precisely vertical direction monitoring. In this article, the authors show two examples on the advantages of using AFM to study the lipid bilayer phase separation. The first part shows that difference in the same lipid bilayers, 1,2-dioleoyl-sn-glycero-3-phosphocholine (DOPC)/sphingomyelin (SM), when different SM sources are introduced. Although no difference would be observed from the optical microscope, the AFM could easily find out the difference. In the second case, the commonly used fluorescence indicator, Texas Red, is introduced in the films. The precise position dispersion of the fluorescence indicator is also probed in AFM study.

\section{Experimental section}

\subsection{Supported membrane synthesis}

The lipids used in this study are DOPC, SM from cow brain (BSM) and SM from egg (ESM); all the lipids and the fluorescence indicator, Texas Red, purchased from Avanti (Alabaster, Al, USA). Antibody of Texas Red, TR-DPPE, is purchased from Molecular Probes (Grand Island, NY, USA). All the chemicals are used without further purification. Lipids' membrane with 1:1 ratio of DOPC to BSM or DOPC to ESM is formed from the deposition of liquid vesicles according to literature. ${ }^{6}$ Single-wall small vesicles are formed from vesicle precursors after 1-min sonication. The supported membrane is prepared on the mica 
Bioinspired, Biomimetic and Nanobiomaterials Volume 3 Issue BBN1
Atomic force microscopic study on lipid bilayer nanoscale phase separation Liu substrate with the incubation of single-wall small vesicles under $50^{\circ} \mathrm{C}$ for $1 \mathrm{~h}$. The rest of the excess unfused vesicles are washed off from the surface. The supported membrane is kept humid with a droplet of water during transportation and measurement.

\subsection{AFM investigation}

Contact mode is used in this study on AFM (Veeco, nanoscope IV, Plainview, NY, USA). The spring index of the AFM tip used is

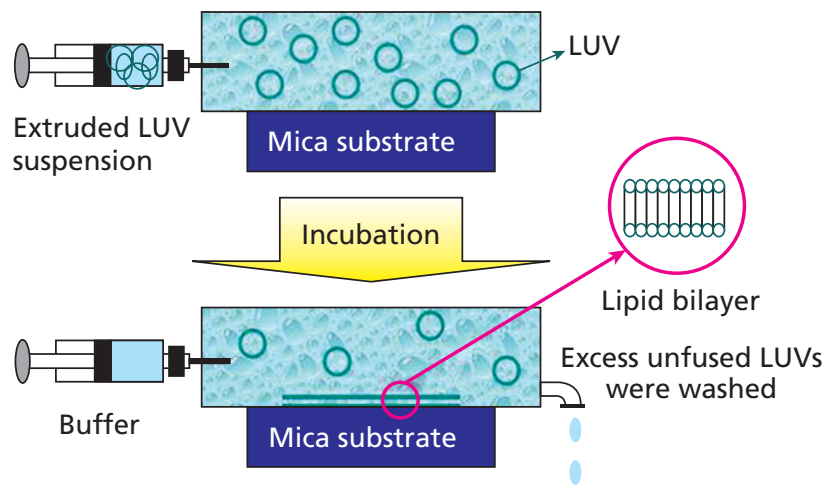

Figure 1. The schematic illumination of the supported lipid membrane preparation
$0 \cdot 32$, and the contact force is about $0 \cdot 3 \mathrm{nN}$. All the experiments are performed at room temperature. The AFM is performed in liquid cell to maintain the lipid bilayer structure from destroying due to solvent evaporation.

\section{Results and discussion}

In this study, the lipid membrane is formed from deposition of large liquid uniform vesicles followed with literature. ${ }^{6}$ After sonication, it is believed that single-wall small vesicles are formed from vesicles precursors. Schematically shown in Figure 1, the supported membrane is prepared on the mica substrate with the incubation of the single-wall small vesicles under $50^{\circ} \mathrm{C}$. Unfused vesicles floating above the supported membrane are flushed away to make the surface clean.

First of all, two different types of DOPC/SM lipid membrane phase separations are investigated by AFM. BSM (from cow brain) and ESM (from eggs) are SM from different sources. ${ }^{9}$ The components in BSM are widely dispersed: $49 \%$ hydrocarbon chains contain 18 carbons; $40 \%$ unsaturated hydrocarbon chains as well as the hydrocarbon chains contain 16-24 carbons; $10 \%$ are other unknown components. The EBM is more uniform with more than $84 \%$ hydrocarbon chain containing 16 carbons and no unsaturated

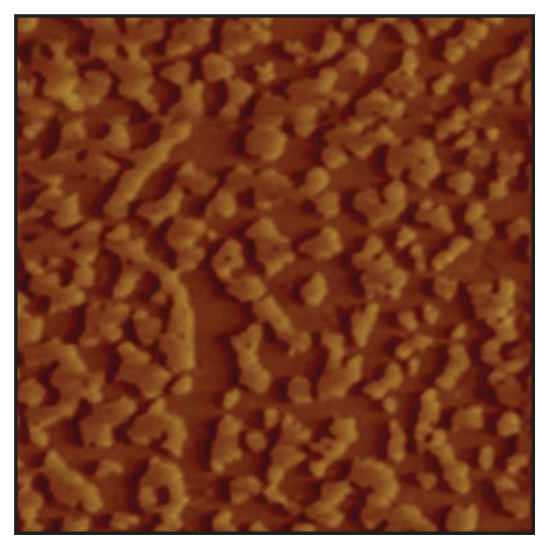

(a)

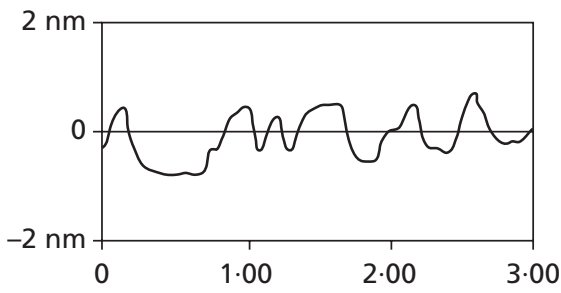

(d)

Figure 2. (a) DOPC/BSM phase separation in $1 \mathrm{~h}$, (b) DOPC/BSM phase separation in $4 \mathrm{~h}$, (c) DOPC/ESM phase separation. The height analysis of $(\mathrm{a}-\mathrm{c})$ are displayed in $(\mathrm{d}-\mathrm{f})$, respectively. Scale size for all the figures are $3 \mu \mathrm{m}$. The white line in $(a-c)$ indicates the height

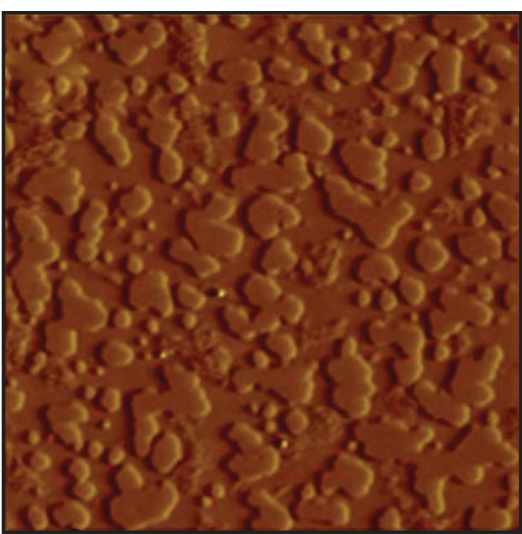

(b)

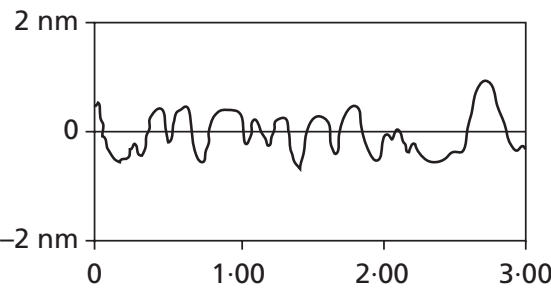

(e)

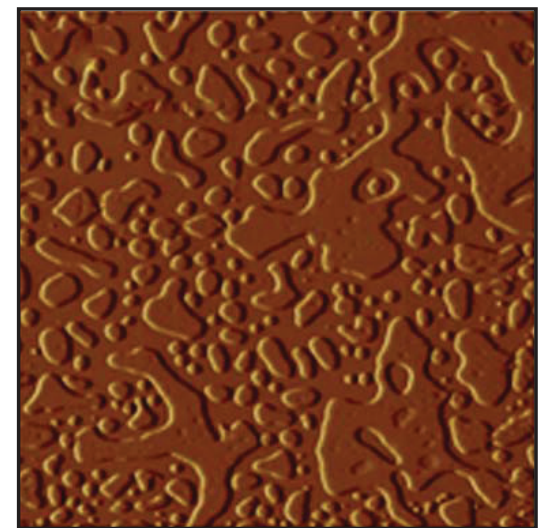

(c)

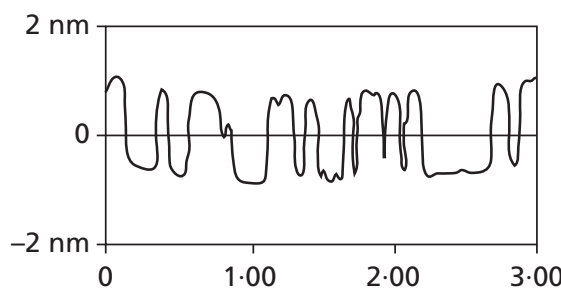

(f)

analysis position in (d-f), respectively. BSM, sphingomyelin from cow brain; DOPC, 1,2-dioleoyl-sn-glycero-3-phosphocholine; ESM, sphingomyelin from egg 
hydrocarbon chains. The variation of the chain's length would contribute to the roughness of the separated domain. Moreover, the rigid unsaturated bonds would decrease the softness of the hydrocarbon chains and occupy more spaces in the membrane, influencing the uniformity of the lipid arrangement. As a result, these differences are obviously observed on the AFM (Figure 2).

As expected, DOPC/BSM phase separation is quite obvious after cooling down in room temperature for $1 \mathrm{~h}$ (Figure 2(a)). The higher domains are BSM, and the other parts are DOPC phase. However, it is a surprise to observe two different BSM domains formed after given enough time for the complete phase separation, one with smooth surface and the other with rough surface. Although the numbers and size are smaller than the smooth domains, the rough BSM domain could also be successfully separated from the DOPC phase consistently, as shown in Figure 2(b). None rough domains are observed on the experiments with DOPC/ESM phase separation (Figure 2(c)).

\begin{tabular}{lccc}
$\begin{array}{l}\text { Carbon number on } \\
\text { lipids chain }\end{array}$ & $L_{\max }: \mathbf{n m}$ & $\begin{array}{c}\Delta\left|L_{\max }-L_{18}\right| \\
: \mathbf{n m}\end{array}$ & $\Delta \mathbf{L}: \mathbf{n m}$ \\
16 & 2.178 & 0.253 & 0.36 \\
18 & 2.431 & 0 & 0 \\
20 & 2.687 & 0.253 & 0.36 \\
22 & 2.937 & 0.506 & 0.72 \\
24 & 3.190 & 0.759 & 1.08 \\
\hline
\end{tabular}

${ }^{a}$ All the data shown in this table are not considered as unsaturated bonds in these lipids.

Table 1. The maximum difference in lipids' length calculated by Tanford equation
Due to the ununiformed BSM components, it is quite reasonable to have separated domains with higher roughness. Suppose that the rougher domains are formed by ununiformed parts of the BSM besides of the largest 18 carbon component, the height variation range could be estimated. According to Tanford equation, which is commonly used to estimate the saturated hydrocarbon chain length (Table 1), the authors could easily calculate the height differences in different lipids ${ }^{10}$ :

1.

$$
L \leq L_{\max }=(0 \cdot 154+0 \cdot 1265 n) \mathrm{nm}
$$

$L_{\max }$ is the maximum height of each lipid with different carbon numbers on the hydrocarbon chains. $\Delta\left|L_{\max }-L_{18}\right|$ is the maximum height difference of the lipids from the one with 18 carbons on the hydrocarbon chains by the calculation. $\Delta L$ shows the final results with correct factor and doubled by considering two lipid layers in the membrane. These $\Delta L$ are matched with the height difference results obtained from AFM. By analyzing the height differences inside of the unsmooth domains, the authors observed three different numbers: $1.05,0.67$ and $0.38 \mathrm{~nm}$. It implies that the AFM study could precisely monitor the phase separation by different lipids. The information obtained from AFM could be much more detailed comparing with the data from optical microscope observation, in which it would be difficult to show the difference of these two lipid bilayer phase separations: DOPC/ $\mathrm{BSM}$ and DOPC/ESM.

Furthermore, Texas Red is introduced into the lipid bilayers in optical microscopic study. It is believed for a long time that the fluorescence indicator molecules dispersed in the domain uniformly since no color intensity differences are observed in optical microscopic images.

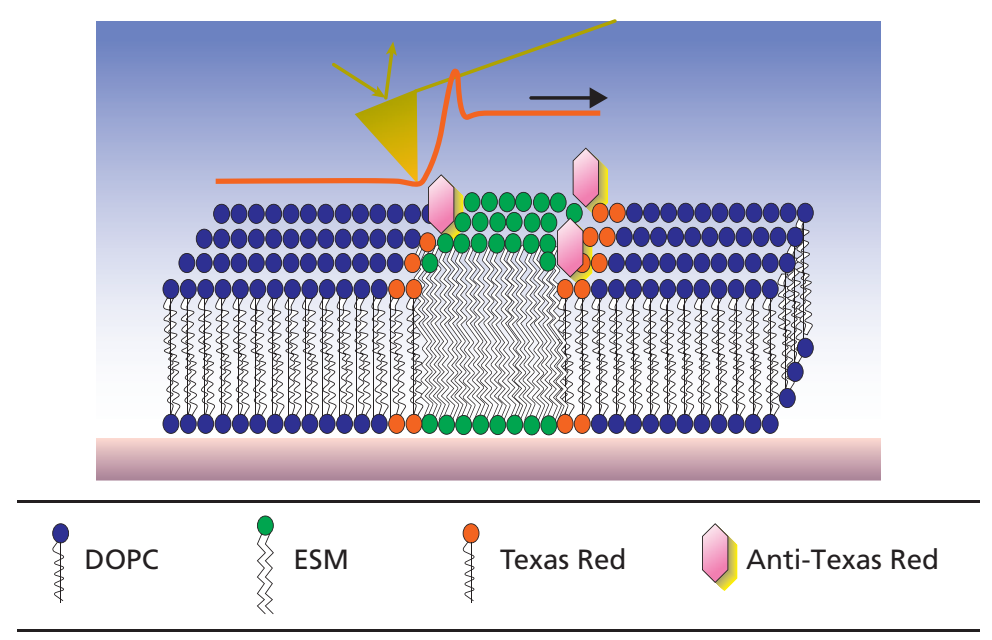

Figure 3. Schematic description of the lipid bilyer phase separation with introduction of fluorescence indicator Texas Red 
Bioinspired, Biomimetic and Nanobiomaterials Volume 3 Issue BBN1
Atomic force microscopic study on lipid bilayer nanoscale phase separation Liu

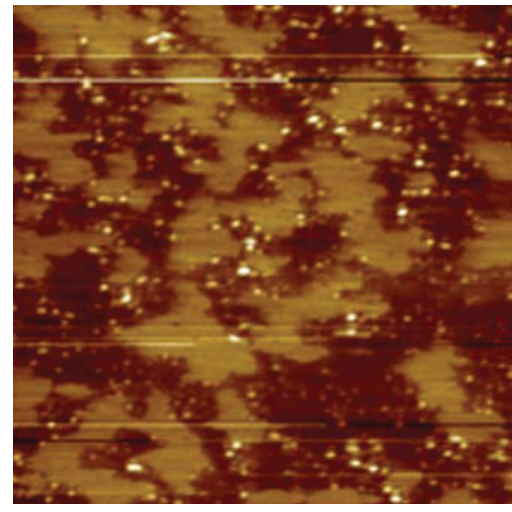

(a)

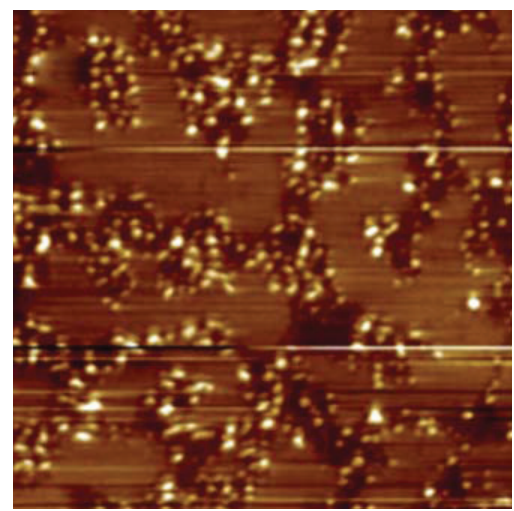

(c)

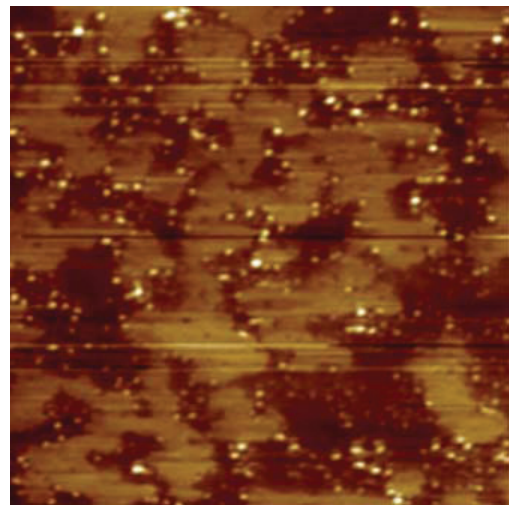

(b)

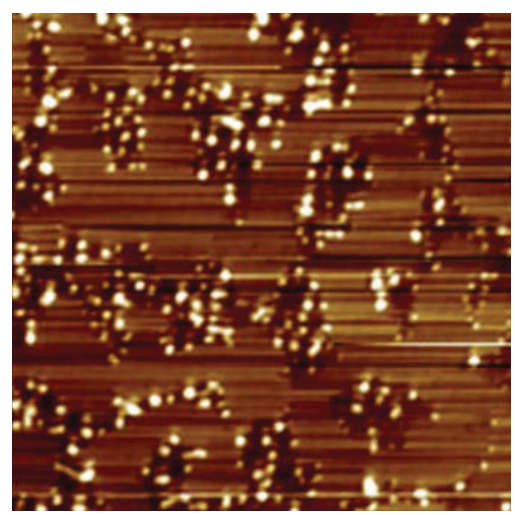

(d)

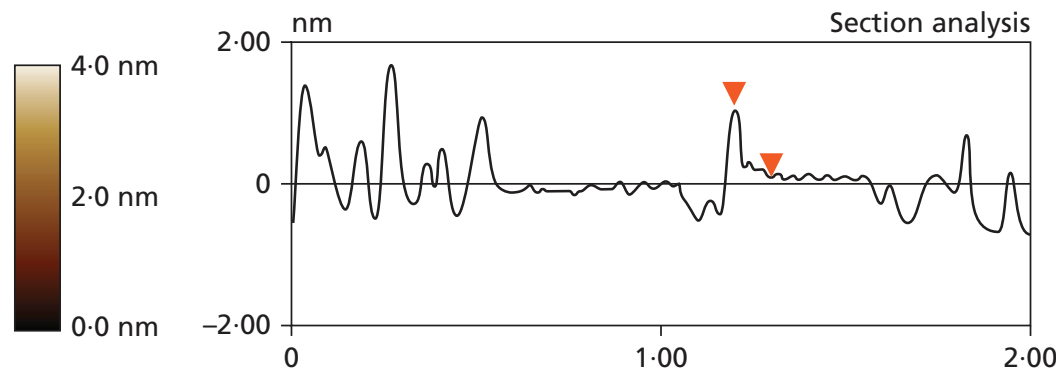

(e)

Figure 4. DOPC/ESM phase separation is studied with introduction of $3 \%$ Texas Red. After adding the antibody of fluorescence indicator: (a) $5 \mathrm{~min}$; (b) $15 \mathrm{~min}$; (c) $48 \mathrm{~min}$; (d) $70 \mathrm{~min}$; (e) the height analysis of

In this study, the authors introduced the antibody of fluorescence indicator after the phase separation and probed the position dispersion of the fluorescence indicator molecules by AFM. The supported membrane is prepared by the same method described earlier, and $3 \%$ of Texas Red is added with DOPC and ESM at the beginning. With such a small per cent of Texas Red, it is assumed that the phase separation behavior would not changed. Texas Red shared the similar tail structure with DOPC but different head structure. Texas Red has a fluorescent head and would be used for fluorescence labeling and preferred to stay in DOPC domains. As known, the Texas Red is used (c). The scan scale of (a-d) are $2 \mu \mathrm{m}$. The height difference between two red arrows in (e) is $1.25 \mathrm{~nm}$. DOPC, 1,2-dioleoyl-sn-glycero-3phosphocholine; ESM, sphingomyelin from egg

different molecules; however, it is hardly to find out the positions of Texas Red by simply AFM scanning and the inadequate height difference compared with DOPC. In order to solve this problem, the antibody of Texas Red is added after phase separation to probe the fluorescence indicator positions. As long as the antibody molecules are added into the liquid cell, they could be closely bonded onto the fluorescence indicator molecules and difficult to lose. With this additional height gained from the antibody molecules, the fluorescence indicator molecules' position would stand up on the lipid bilayers and very ease to be monitored. 
Only $5 \mathrm{~min}$ after the addition of antibody molecules into the phase separated DOPC/ESM bilayer, a few white dots appeared in DOPC phase boundary close to the ESM phase. The height is around $1-2 \mathrm{~nm}$, which is consistent with one lipid molecule (Figure 4(e)). This change is only performed when the antibody molecules are introduced, which implies that the additional height detection by AFM is due to the close bonding of antibody molecules and fluorescence indicator molecules. More white dots are detected with longer reaction time, and some fluorescence indicator molecules begin to show up inside the DOPC domain. Meanwhile, no white dots presented fluorescence indicator antibody molecules when antibody molecules are added onto the DOPC/ESM bilayer without fluorescence indicator molecules or pure ESM bilayer without fluorescence indicator molecules.

It is first of all implied that the fluorescence indicator molecules are accumulated in DOPC phase, which is consistent with the literatures. ${ }^{8}$ And the missing details from the previous study are provided now by AFM. Assuming that the selectivity of the antibody molecules is equal to all the fluorescence indicator molecules, it would be reasonable to draw the conclusion that the concentration of the fluorescence indicator molecules is higher at the boundary and lower inside the DOPC domains. There are two possible explanations for this interesting finding. One would be the fluorescence indicator molecules used to stay in closely packed ESM solid phase are repulsed out and stay on the boundary due to the limitation of the mobility inside the supported membrane. The other one would be that lipid arrangement is less ordered at the boundary, where the possibility of stabilizing the 'impurity' fluorescence indicator molecules is higher than the more ordered position inside the domains.

\section{Conclusion}

In this study, AFM is used to systematically investigate the nanoscale phase separation of DOPC/BSM and DOPC/ESM liquid bilayers as the supported membrane. The different phase separation behavior of DOPC/BSM and DOPC/ESM liquid bilayers are observed and analyzed by AFM. Also, AFM is used to probe the position of the fluorescence indicator, which is commonly used in the phase separation observation in optical microscope. The fluorescence indicator is found to be preferred on the boundary of the domains instead of the uniform dispersion. AFM study provided more details on the lipid phase separation, which would be considered as a powerful method when deep understanding of the systems is required.

\section{REFERENCES}

1. Binder, W. H.; Barragan, V.; Menger, F. M. Angewandte Chemie International Edition 2003, 42, 5802-5827.

2. Simons, K.; Toomre, D. Nature Reviews Molecular Cell Biology 2000, 1, 31-39.

3. Simons, K.; Ikonen, E. Nature 1997, 387, 569-572.
4. Wei, T.; Chi, M.; Tsai, C.; Ko, H.; Chen, J. Langmuir 2013, 29, 9972-9978.

5. Forney, B.; Baguenard, C.; Guymon, C. Chemistry of Materials 2013, 25, 2950-2960.

6. Brian, A. A.; McConnell, H. M. Proceedings of the National Academy of Sciences of the United States of America 1984, 81, 6159-6163.

7. Giocondi, M.; Vie', V.; Lesniewska, E.; Milhiet, P.; ZinkeAllmang, M.; Grimellec, C. Langmuir 2001, 17, 1653-1659.

8. Li, L.; Liang, X. Y.; Lin, M. Y.; Qiu, F.; Yang, Y. L. Journal of the American Chemical Society 2005, 127, 17996-17997.

9. BSM and ESM. See http://www.avantilipids.com/index. php?option=com_content $\&$ view $=$ article $\&$ id $=436 \&$ Itemid $=$ 277\&catnumber $=860062$ and http://www.avantilipids.com/ index.php?option=com_content $\&$ view=article $\&$ id $=437 \&$ Item $\mathrm{id}=277$ \& catnumber $=860061$ for further deatils.

10. Tanford, C. The Hydrophobic Effect. New York: Wiley, 1980.

\section{WHAT DO YOU THINK?}

To discuss this paper, please email up to 500 words to the managing editor at bbn@icepublishing.com

Your contribution will be forwarded to the author(s) for a reply and, if considered appropriate by the editor-inchief, will be published as a discussion in a future issue of the journal.

ICE Science journals rely entirely on contributions sent in by professionals, academics and students coming from the field of materials science and engineering. Articles should be within 5000-7000 words long (short communications and opinion articles should be within 2000 words long), with adequate illustrations and references. To access our author guidelines and how to submit your paper, please refer to the journal website at www.icevirtuallibrary.com/bbn 